\title{
MEDICAL SCIENCE
}

\section{Safety and efficacy of xenon in routine use as an inhalational anaesthetic}

\author{
BURKHARD LACHMANN SIEGFRIED ARMBRUSTER \\ WOLFGANG SCHAIRER MARGREET LANDSTRA \\ ADRIANUS TROUWBORST GEERT-JAN VAN DAAL ARIE KUSUMA \\ WILHELM ERDMANN
}

40 patients ( 24 male, 16 female, aged $21-59$ years) of American Society of Anesthesiologists class I or II who were undergoing routine surgery took part in a randomised, double-blind comparison of the anaesthetic efficacy and potency of xenon and nitrous oxide and their effects on the circulatory and respiratory systems. During anaesthesia, for each rise in blood pressure of more than $20 \%$ of the preanaesthetic (baseline) value, the patient received $0.1 \mathrm{mg}$ fentanyl. The total amount of fentanyl required per patient was used as an index of the anaesthetic potency of the study gases. Patients in the xenon group required on average only $0.05 \mathrm{mg}$ fentanyl, whereas those in the nitrous oxide group required $0.24 \mathrm{mg}$ fentanyl; the duration of anaesthesia was similar in the two groups. Changes in blood pressure were significantly greater throughout the study in the nitrous oxide than in the xenon group. Thorax-lung compliance fell during the study period in the nitrous oxide group but not in the xenon group. Thus, xenon is a potent and effective anaesthetic which can be safely used under routine conditions. Lancet 1990; 335: 1413-15.

\section{Introduction}

The anaesthetic gases and vapours in routine use have undesirable side-effects on both patients and medical and nursing staff and are a source of pollution of the environment.1-6 The search continues, therefore, for a gas that meets all the requirements for an anaesthetic agent but has none of these disadvantages. Xenon offers many advantages over routinely used gases. ${ }^{7}$ It is non-explosive; probably does not undergo biotransformation; is non-toxic; and offers rapid induction of and recovery from anaesthesia. ${ }^{8-11}$ In experimental studies we have measured tooth-pulp evoked potentials to evaluate pain $^{12,13}$ and confirmed that the analgesic efficacy of xenon is superior to that of nitrous oxide at the same concentration $(70 \%) ; ;^{14}$ xenon had no effect on systemic haemodynamics or local organ perfusion ${ }^{15}$ as measured by means of radionuclidelabelled microspheres. ${ }^{16,17}$

The main reason why xenon is not routinely used is that there have been no commercially available anaesthesia machines in which to use the gas, which is more expensive than nitrous oxide and the volatile anaesthetics. There has been very limited clinical experience with xenon, ${ }^{8-11}$ and no studies have compared its anaesthetic efficacy and effects on haemodynamics and lung mechanics with those of the "gold standard", nitrous oxide. We have investigated the anaesthetic efficacy of a mixture of $70 \%$ xenon $/ 30 \%$ oxygen and $70 \%$ nitrous oxide $30 \%$ oxygen, incorporated in a balanced anaesthetic method, in a randomised double-blind study during routine surgery.

Before induction of anaesthesia, baseline blood pressure levels were established for each patient. In accordance with the balanced anaesthesia method, rises in blood pressure of more than $20 \%$ of the baseline value were treated with $0 \cdot 1$ mg fentanyl intravenously. The total amount of fentanyl required for each patient was taken as an objective index of the potency and overall efficacy of the two gases.

\section{Patients and methods}

40 patients (24 men, 16 women; aged 21-59 years, American Society of Anesthesiologists [ASA] class I or II) undergoing routine operations of similar durations gave written informed consent to take part in the study. The study protocol was approved by the hospital medical ethics review committee. The patients were randomised as follows: plastic surgery to face, breast, or genitals 2 to xenon, 4 to nitrous oxide; intraperitoneal procedures 3 to xenon, 2 to nitrous oxide; bloodless surgery of limbs 8 to xenon, 7 to nitrous oxide; other orthopaedic procedures 7 to each group.

All patients received methylatropine bromide $(0.002 \mathrm{mg} / \mathrm{kg}$ intravenously) as premedication. After preoxygenation (with pure oxygen) for about $5 \mathrm{~min}$ (partial denitrogenation by way of a face mask), anaesthesia was induced with intravenous thiopentone (5-7 $\mathrm{mg} / \mathrm{kg})$ and pancuronium bromide $(0.1 \mathrm{mg} / \mathrm{kg})$, then endotracheal intubation was carried out. Further denitrogenation was brought about by ventilation with pure oxygen for about $6-8 \mathrm{~min}$. The anaesthetic gas mixture, either xenon/oxygen (70/30) or nitrous oxide/oxygen (70/30), was administered by means of a closedcircuit anaesthesia machine (a combination of a 'Servoventilator $900 c$ ' with a modified '985 Anesthesia Circle', Siemens Elema, Solna, Sweden). To ensure double-blinding, the anaesthetic gases

ADDRESSES: Department of Anaesthesiology, University Hospital Dijkzigt, Erasmus University Rotterdam, The Netherlands (Prof B. Lachmann, MD, S. Armbruster, W. Schairer, M. Landstra, A Trouwborst, MD, G-J van Daal, A. Kusuma, MD, Prof W. Erdmann, MD). Correspondence to Prof B. Lachmann, Department of Anaesthesiology, Erasmus University, Postbox 1738 (Room EE2393) 3000 DR Rotterdam, The Netherlands 
TABLE I-CLINICAL DATA FOR STUDY GROUPS

\begin{tabular}{l|c|c}
\hline \multirow{2}{*}{-} & \multicolumn{2}{|c}{ Mean (SD) or number of patients } \\
\cline { 2 - 3 } & $\begin{array}{c}\text { Xenon } \\
(\mathrm{n}=20)\end{array}$ & $\begin{array}{c}\text { Nitrous oxide } \\
(\mathrm{n}=20)\end{array}$ \\
\hline Age $(\mathrm{yr})$ & $37(10)$ & $33(10)$ \\
Weight $(\mathrm{kg})$ & $71(14)$ & $73(11)$ \\
Helght $(\mathrm{cm})$ & $172(9)$ & $175(8)$ \\
Male/Female & $12 / 8$ & $12 / 8$ \\
ASA & 17 & 16 \\
Class I & 3 & 4 \\
Class II & & \\
Duration (min) of & $73(37)$ & $69(33)$ \\
$\quad$ Closed-circuit anaesthesia & $52(33)$ & $54(31)$ \\
Surgery & $0.05(0.08)$ & $0.224(0 \cdot 16)$ \\
Fentanyl (mg/patient) &
\end{tabular}

were administered by anaesthetists other than those responsible for patient treatment and data collection. Minute ventilation was set to maintain end-tidal carbon dioxide at 4.0-4.5 vol $\%$. After wash-in of the study gas to about $70 \%$, the fresh gas flow was adjusted to maintain an inspired oxygen concentration of about $30 \%$; the surgical procedure was then started. For each patient, if the blood pressure rose by more than $20 \%$ of the baseline value, intravenous fentanyl $(0.1 \mathrm{mg})$ was given. Muscle relaxation was monitored by means of a 'Relaxograph' (Datex Medical, Finland) and additional doses of pancuronium bromide were given as required. At the end of the surgical procedure residual muscle relaxation was antagonised by methylatropine bromide $(0.035 \mathrm{mg} / \mathrm{kg})$ and neostigmine $(0.04 \mathrm{mg} / \mathrm{kg})$ and the study gas was washed out by ventilation with pure oxygen. The time from withdrawal of the study gas to return of consciousness was recorded and all patients were interviewed after $30 \mathrm{~min}$ and after $24 \mathrm{~h}$ to evaluate peroperative awareness and general patient acceptability postoperatively.

At baseline (after wash-in of the study gas to $70 \%$ ) and continuously throughout the operation we monitored vital measurements including heart rate, non-invasive systolic/diastolic blood pressure (measured every $3 \mathrm{~min}$ ) (Marquette monitor series 7000), arterial saturation (N-100 'Pulsoximeter', Nellcor, USA), end-tidal carbon dioxide (analyser 930, Siemens Elema); and lung mechanical measurements including thorax-lung compliance (calculated from the ratio of tidal volume and the corresponding change in elastic static pressure) and expiratory airway resistance (the ratio of driving pressure [end-inspiratory pause pressure minus expiratory airway pressure] and initial expiratory flow; Lung Mechanics Calculator 940, Siemens Elema, Sweden). The lung mechanics calculator is a reliable and accurate measure of lung mechanics. ${ }^{18}$ All these measurements were automatically stored once a minute (as available) on-line by means of an IBM-compatible personal computer.

During anaesthesia the closed-circuit system was flushed with fresh gas every $20 \mathrm{~min}$ to avoid accumulation of nitrogen. Ventilation with one or two sighs was applied if oxygen saturation fell below $95 \%$ at an inspired oxygen concentration of $30 \%$.
To avoid excessive influence by individual variability, baseline values of physiological measurements were set at $100 \%$; further analysis was made on the percentage values at the specified times. Group differences in gender and ASA classification were analysed by the Kruskal-Wallis test and those in age, weight, and height by the chi-square test. All other variables were analysed by the Mann-Whitney-Wilcoxon test for between-group comparison or the sign test for paired samples for within-group comparison. All tests of statistical significance were two-tailed and data were tested at the 0.05 significance level. All values are presented as mean and standard deviation.

\section{Results}

The two study groups were comparable in age, weight, height, sex, ASA classification, duration of closed-circuit anaesthesia, and duration of surgery (table I). However, the average amount of fentanyl required was about five times greater $(\mathrm{p}<0.001)$ in the nitrous oxide group than in the xenon group (table $\mathrm{II}) .13$ of the $20(65 \%)$ xenon-treated patients required no fentanyl compared with only 1 patient $(5 \%)$ in the nitrous oxide group (table III). Changes in systolic blood pressure throughout the operations were significantly smaller $(p<0.01)$ in the xenon group than in the nitrous oxide group (see accompanying figure). Diastolic blood pressure and pulse measurements are shown in table II.

Peripheral arterial oxygenation was sufficient in both groups with an inspired oxygen concentration of about $30 \%$, and there was no difference in oxygen saturation between the groups at the end of the operation. However, in 8 nitrous-oxide-treated patients oxygen saturation fell below $92 \%$ during the first 20 min after induction; at the same time there was also a fall in thorax-lung compliance of more than $20 \%$. After treatment with one or two sighs with a double tidal volume, thorax-lung compliance was partly restored and oxygen saturation rose to about $96 \%$. The total number of sighs was significantly higher in the nitrous oxide $(n=43)$ than in the xenon group $(n=6)$, but thorax-lung compliance was significantly lower in the nitrous oxide group at the end of anaesthesia than at baseline $(84[10.5] \%)$ and than in the xenon group at the end of anaesthesia (97.5 [7.6]\%). Expiratory lung resistance was significantly higher in both groups at the end of anaesthesia (xenon $116[19] \%$; nitrous oxide $129[48] \%$ ) than at baseline but the difference between the groups was not significant.

Recovery time from anaesthesia was similar in both groups (xenon 4 [3] min; nitrous oxide 7 [4] min). Only the 5 patients in the nitrous oxide group who received more than $0.4 \mathrm{mg}$ fentanyl had long recovery times and took longer to orientate themselves clearly. No patient complained of

TABLE II-MEAN (SD) PERCENTAGE CHANGES FROM BASELINE VALUES IN CIRCULATORY MEASUREMENTS RELATED TO FENTANYL DOSE

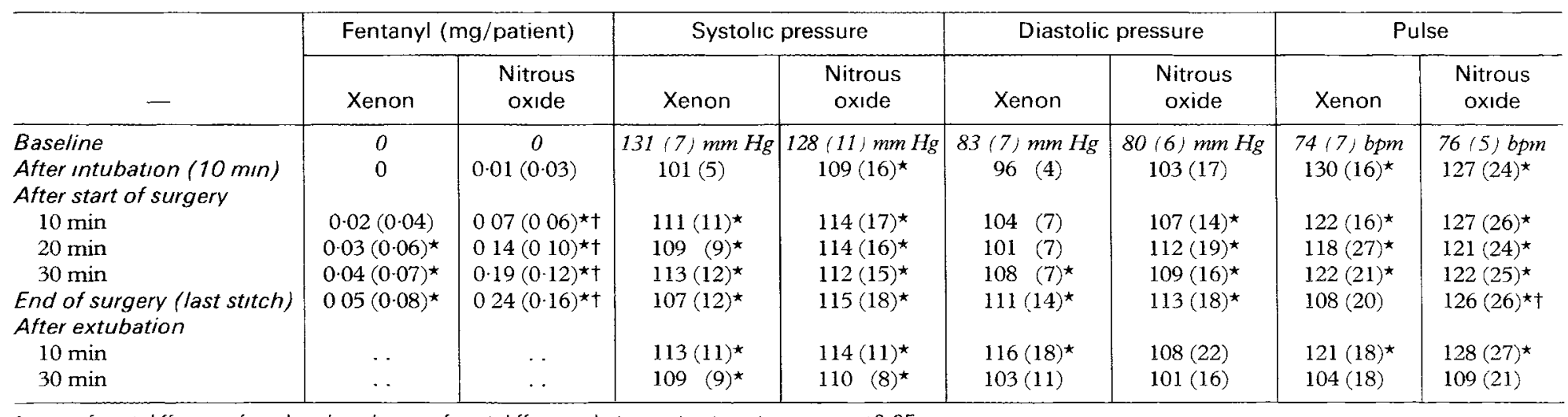

- = significant difference from baseline, $\uparrow=$ significant difference between treatment groups, $p<005$ 
TABLE III-AMOUNT OF FENTANYL REQUIRED PER PATIENT

\begin{tabular}{c|c|c}
\hline \multirow{2}{*}{ Fentanyl (mg/patient) } & \multicolumn{2}{|c}{ Number of patients } \\
\cline { 2 - 3 } & Xenon & Nitrous oxide \\
\hline 0 & 13 & 1 \\
0.1 & 5 & 8 \\
02 & 1 & 2 \\
0.3 & 1 & 4 \\
04 & 0 & 3 \\
0.5 & 0 & 1 \\
0.6 & 0 & 1 \\
\hline
\end{tabular}

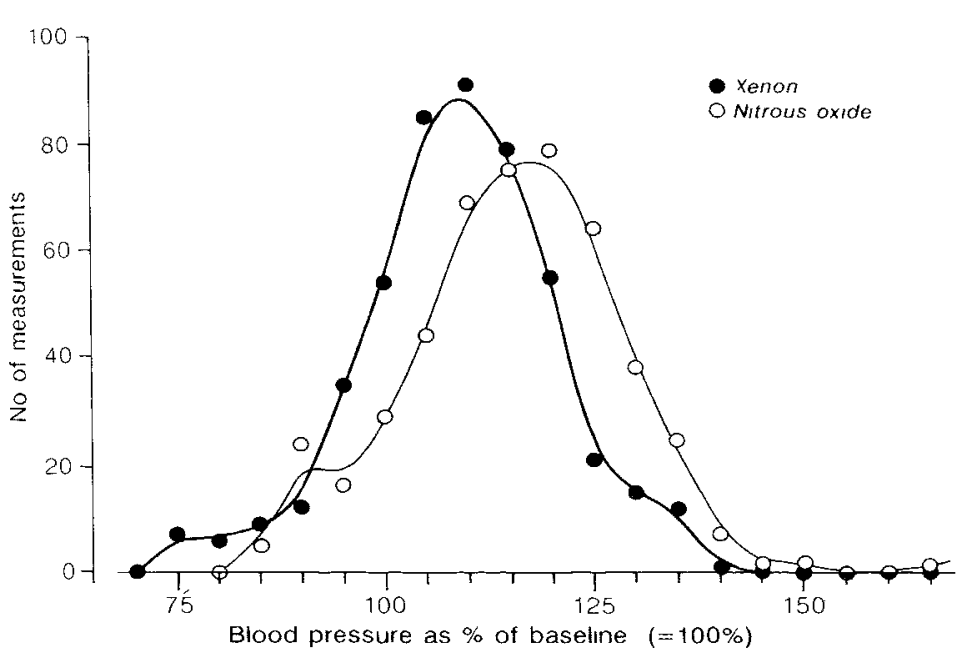

Distribution of all measured systolic blood-pressure changes from baseline values during anaesthesia.

peroperative awareness. Patients treated with xenon, however, reported "nice feelings and pleasant dreams", without being able to remember any details. The anaesthetic course of all forty procedures was uneventful, as was the postoperative period.

\section{Discussion}

This study shows that a concentration of $70 \%$ xenon results in satisfactory anaesthesia for surgical procedures. The requirement for fentanyl in the xenon group was only a fifth of that in the nitrous oxide group and $95 \%$ of the patients treated with nitrous oxide required fentanyl compared with only $35 \%$ in the xenon-treated group. The lower opioid consumption during xenon anaesthesia, combined with the very fast elimination of xenon, may explain the faster recovery after xenon than after nitrous oxide.

With both anaesthetic regimens blood pressure and heart rate were adequately controlled. The overall smaller rise in blood pressure in the xenon group than in the nitrous oxide group indicates more stable cardiocirculatory conditions as a result of less stress-induced sympathetic stimulation; this was confirmed by the finding that adrenaline levels did not change during the operation in the xenon group only. ${ }^{19}$ The difference may be due to the more potent analgesic effect (peripherally and/or centrally) of xenon.

Oxygen saturation fell below $92 \%$ in 8 patients in the nitrous oxide group but in no patient in the xenon group. Nitrous oxide is more soluble than xenon, which could lead to absorptive atelectasis. This explanation is supported by the finding that thorax-lung compliance fell at the same time, but it remains speculative because we did not measure functional residual capacity in these patients at the same time. There was only slight deterioration in lung mechanics during xenon anaesthesia, which suggests that xenon can be used safely in older patients and those with chronic lung diseases.
The absence of cardiovascular side-effects, the lack of reduction in local organ perfusion (especially in heart and brain), ${ }^{15}$ and the established potency as an inhaled anaesthetic and analgesic ${ }^{\mathbf{1 4}}$ make xenon a very promising anaesthetic agent for routine use. Xenon does not present the problems and hazards to patients, medical and nursing staff, and the environment that nitrous oxide and other volatile anaesthetics do. The average amount of xenon required per anaesthetic procedure was 10 litres; with the development of closed-circuit anaesthesia systems, costs of routine xenon use can be reduced and, therefore, will not differ substantially from those of other anaesthetic procedures.

We thank our surgical and nursing colleagues for their cooperation; Siemens Elema, Sweden, who provided machines and gases; the Dutch Foundation for Medical Research (SFMO) who supported the study with grants; and Dr S. G. Olsson (Siemens Elema) and Dr J. Gesing (Kreiskrankenhaus Crailsheim) for valuable discussions and advice in planning the study.

\section{REFERENCES}

1. Amess JAL, Rees GM, Burman JF, Nancekievill DG, Mollin DL. Megaloblastic haemopoiesis in patients receiving nitrous oxide. Lancet 1978; ii: $339-42$.

2. Layzer RB. Myeloneuropathy after prolonged exposure to nitrous oxide. Lancet 1978; ii: $1227-30$.

3. Lane GA, Nahrwold ML, Tait AR, Taylor-Busch M, Cohen PJ. Anesthetics as teratogens: nitrous oxide is fetotoxic, xenon is not. Science 1980; 210: 899-901.

4. Spence AA. Environmental pollution by inhalation anaesthetics. $\mathrm{Br} \mathcal{F}$ Anaesth 1987; 59: 96-103.

5. Cohen EN, Bellville JW, Brown BW. Anesthesia pregnancy and miscarriage: a study of operating room nurses and anesthesists. Anesthesiology 1971; 35:343-47.

6. Dyck PJ, Grina LA, Edward BM, et al. Nitrous oxide neurotoxicity studies in man and rat. Anesthesiology 1980; 53: 205-09.

7. Lawrence JH, Loomis WF, Tobias CA, Turpin FH. Preliminary observations on the narcotic effect of xenon with a review of values for solubilities of gases in water and oils. $\mathcal{F}$ Physzol 1946; 105: 197-204.

8. Cullen SC, Gross EG. The anesthetic properties of xenon in animals and human beings with additional observations on krypton. Science 1951; 113: $580-82$.

9. Pittinger $\mathrm{CB}$, Moyers J, Cullen SC, Featherstone RM, Gross EG. Clinico-pathologic studies associated with xenon anesthesia. Anesthesiology 1953; 14: 10-17.

10. Morris LE, Knott JR, Pittinger CB. Electro-encephalic and blood gas observations in human surgical patients during xenon anesthesia. Anesthesiology 1955; 16: 312-19.

11. Cullen SC, Eger EI, Cullen BF, Gregory P. Observations on the anesthetic effect of the combination of xenon and halothane. Anesthesiology 1969; 31: 305-09.

12. Chudler EH, Dong WK. The assessment of pain by cerebral evoked potentials. Pain 1983; 16: 221-24.

13. Fernades de Lima VM, Chatrian GE, Lettich E, Canfield RC, Miller $\mathrm{RC}$, Soso MJ. Electrical stimulation of tooth pulp in humans. Pain 1982; 14: 207-32.

14. I achmann B, Trouwborst A, Schairer W, Armbruster S, Erdmann W. Xenon anesthesia and its analgesic effects. In: abstr vol 1. 9th World Congress on Anaesthetics: Washington, USA, 1988: A0079.

15. Lachmann B, Verdouw PD, Scharrer W, van Woerkens LJ, van Daal GJ Xenon anesthesia and circulation. In: Abstr vol 1.9th World Congress on Anaesthetics: Washington, USA, 1988: A0242.

16. Heymann MA, Payne BD, Hoffman JIE, Rudolph AM. Blood flow measurements with radionuclide-labelled particles. Prog Cardiovasc Dis 1977; 20: 55-79.

17. Saxena PR, Verdouw PD. 5-Carboxamide trytapine, a compound with high affinity for 5-hydroxyrtryptamine, binding sites, dilates arterioles and constricts arteriovenous anastomoses. Br $\mathcal{F}$ Pharmacol 1985; 84: $533-44$

18. Jonson B, Nordstrom L, Olsson SG, Akerback D. Monitoring of lung mechanics during automatic ventilation. A new device. Bull Physiopath Resp 1975; 11: $729-43$.

19. Boomsma F, Rupreht J, Man in 't Veld AJ, de Jong FH, Dzoljic M, I achmann B. Haemodynamic and neurohumoral effects of xenon anaesthesia. A comparison with nitrous oxide. Anaesthesia 1990; 45: $273-78$. 